\title{
Atomistic simulation of ion solvation in water explains surface preference of halides
}

\author{
Carl Calemann ${ }^{\mathrm{a}, 1}$, Jochen S. Hub ${ }^{\mathrm{b}, 1}$, Paul J. van Maaren ${ }^{\mathrm{b}}$, and David van der Spoel $\mathrm{b}^{\mathrm{b}, 2}$ \\ ${ }^{a}$ Center for Free-Electron Laser Science, Deutsches Elektronen-Synchrotron, Notkestraße 85, DE-22607 Hamburg, Germany; and ${ }^{b}$ Department of Cell and \\ Molecular Biology, Biomedical Center, Box 596, Uppsala University, SE-75 124 Uppsala, Sweden
}

Edited* by Michael Levitt, Stanford University School of Medicine, Stanford, CA, and approved March 7, 2011 (received for review December 1, 2010)

\begin{abstract}
Water is a demanding partner. It strongly attracts ions, yet some halide anions-chloride, bromide, and iodide-are expelled to the air/water interface. This has important implications for chemistry in the atmosphere, including the ozone cycle. We present a quantitative analysis of the energetics of ion solvation based on molecular simulations of all stable alkali and halide ions in water droplets. The potentials of mean force for $\mathrm{Cl}^{-}, \mathrm{Br}^{-}$, and $\mathrm{I}^{-}$have shallow minima near the surface. We demonstrate that these minima derive from more favorable water-water interaction energy when the ions are partially desolvated. Alkali cations are on the inside because of the favorable ion-water energy, whereas $F^{-}$is driven inside by entropy. Models attempting to explain the surface preference based on one or more ion properties such as polarizability or size are shown to lead to qualitative and quantitative errors, prompting a paradigm shift in chemistry away from such simplifications.
\end{abstract}

ozone layer | GROMACS | aerosol | thermodynamics

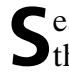
a water becomes airborne in the form of droplets formed at the sea surface by the action of waves (1). These droplets can be carried by the wind, and they interact with other constituents of the atmosphere (Fig. 1A). On the surface of the small water droplets, halide anions can be converted into halogen atoms by absorption of light $(2,3)$, and the air-water interface serves to increase certain reaction probabilities $(4,5)$. One example is the oxidation of $\mathrm{Cl}^{-}$and $\mathrm{Br}^{-}$by $\mathrm{OH}$ radicals or $\mathrm{O}_{3}$ that can occur at the air-water interface, with mechanisms different from those in the bulk phase (6), leading to natural ozone depletion in the stratosphere.

Both theoretical and experimental studies have shown that $\mathrm{Cl}^{-}, \mathrm{Br}^{-}$and $\mathrm{I}^{-}$prefer to be at the surface of water droplets (Fig. $1 A$ ), whereas $\mathrm{F}^{-}$and alkali cations strive to become fully solvated in the bulk of water droplets (7-12). The $\mathrm{Br}^{-}$concentration is enhanced more at the surface than the $\mathrm{Cl}^{-}$concentration (13), an effect that may be enhanced in mixtures containing both $\mathrm{Br}^{-}$and $\mathrm{Cl}^{-}$, such as seawater (14). Because $\mathrm{Br}^{-}$is more reactive than $\mathrm{Cl}^{-}$, the surface preference has an impact on the chemistry in the atmosphere, despite the fact that the content of $\mathrm{Cl}^{-}$in bulk sea water is about three orders of magnitude higher than that of $\mathrm{Br}^{-}$(15). The energetic and structural mechanisms underlying the surface preference of large halide anions have been studied in quite some detail (16-18), but the phenomenon is still not understood quantitatively (10). Only very recently has the surface absorption free energy of one halide anion, $\mathrm{Br}^{-}$, been determined experimentally (19). Recent progress in development of force fields for water (20) and ions (21) finally allows establishment of the surface preferences for all halide and alkali ions quantitatively.

Here we present the energetics of ion solvation in a water droplet with a radius of approximately $1.1 \mathrm{~nm}$ for all stable halide anions $\left(\mathrm{F}^{-}, \mathrm{Cl}^{-}, \mathrm{Br}^{-}, \mathrm{I}^{-}\right)$and alkali cations $\left(\mathrm{Li}^{+}, \mathrm{Na}^{+}, \mathrm{K}^{+}\right.$, $\mathrm{Rb}^{+}, \mathrm{Cs}^{+}$). Potentials of mean force (PMFs) (also referred to as free-energy profiles $\Delta G(r))$ at room temperature $(293.15 \mathrm{~K})$ were derived from molecular dynamics simulations using polarizable water and ion models. We decompose the PMFs into enthalpic and entropic components. In a second step, the enthalpic component is divided into contributions from ion-water and waterwater interactions. This procedure allows one to identify the driving forces governing the surface/bulk preference.

\section{Results and Discussion}

Fig. $1 B$ and $C$ presents potentials of mean force $\Delta G(r)$ for the ion solvation into the water droplet, where $r$ denotes the distance of the ion from the center of mass of the water droplet, as illustrated in Fig. 1A. The gray dashed lines in the figures indicate the Gibbs dividing surface of a water droplet with no ion. The surface of the droplets are not at all smooth, especially not when an ion is being dragged out from the droplet (as can be seen in Fig. 2), and the Gibbs dividing surface should therefore be seen as an approximation only. The PMFs were defined to be zero in the droplet interior, and the flat regions at $r>2.5 \mathrm{~nm}$ correspond to the fully desolvated ions and thus indicate the complete desolvation free energy. The insets of Fig. $1 B$ and $C$ show a close-up of the PMF, revealing local minima near the droplet surface in the PMFs for $\mathrm{Cl}^{-}, \mathrm{Br}^{-}$, and $\mathrm{I}^{-}$(Fig. $1 B$ ). The surface preference as measured from the depth of the minimum increases with the size of ion but only for the halide anions.

The PMFs can be used to compute concentration enhancement in the outermost water layer $(0.75 \mathrm{~nm} \leq r \leq 1.1 \mathrm{~nm})$. For $\mathrm{Cl}^{-}$the surface concentration will be 1.6 times higher than in the bulk, for $\mathrm{Br}^{-}$and $\mathrm{I}^{-}$the factors are 7 and 13, respectively (see Methods in SI Appendix). Thus, $\mathrm{I}^{-}$is enhanced twice as strong on the surface than $\mathrm{Br}^{-}$, in qualitative agreement with experimental findings (9). Comparing the individual concentration enhancement factors to experiments requires incorporation of the probe depth of the specific experiment (10). Assuming an exponentially decaying probe signal with decay length between 0.5 and $1 \mathrm{~nm}$, the PMFs would imply that experiments measure concentration enhancements by factors of approximately 1,4 , and 6 , for $\mathrm{Cl}^{-}$, $\mathrm{Br}^{-}$, and $\mathrm{I}^{-}$, respectively (Methods in SI Appendix). Hence, the slight concentration enhancement of $\mathrm{Cl}^{-}$may not be visible in such experiments. The value for $\mathrm{Br}^{-}$would translate into a surface affinity of approximately $3 \mathrm{~kJ} / \mathrm{mol}$, in excellent agreement with recent spectroscopic experiments (19). In contrast to the large halide ions, the PMFs for $\mathrm{F}^{-}$as well as for the alkali cations Fig. $1 C$ Inset) monotonically increase with $r$, demonstrating the bulk preference of these ions, in accord with previous observations $(12,22-24)$. Now that we have established quantitative correspondence of our calculations with available data, we can tackle the question of why the ions behave like they do.

Author contributions: C.C., J.S.H., P.J.v.M., and D.v.d.S. designed research; C.C. and J.S.H. performed research; C.C. and J.S.H. analyzed data; and C.C., J.S.H., and D.v.d.S. wrote the paper.

The authors declare no conflict of interest.

*This Direct Submission article had a prearranged editor.

${ }^{1}$ C.C. and J.S.H. contributed equally to this work.

${ }^{2}$ To whom correspondence should be addressed. E-mail: spoel@xray.bmc.uu.se.

This article contains supporting information online at www.pnas.org/lookup/suppl/ doi:10.1073/pnas.1017903108/-/DCSupplemental. 

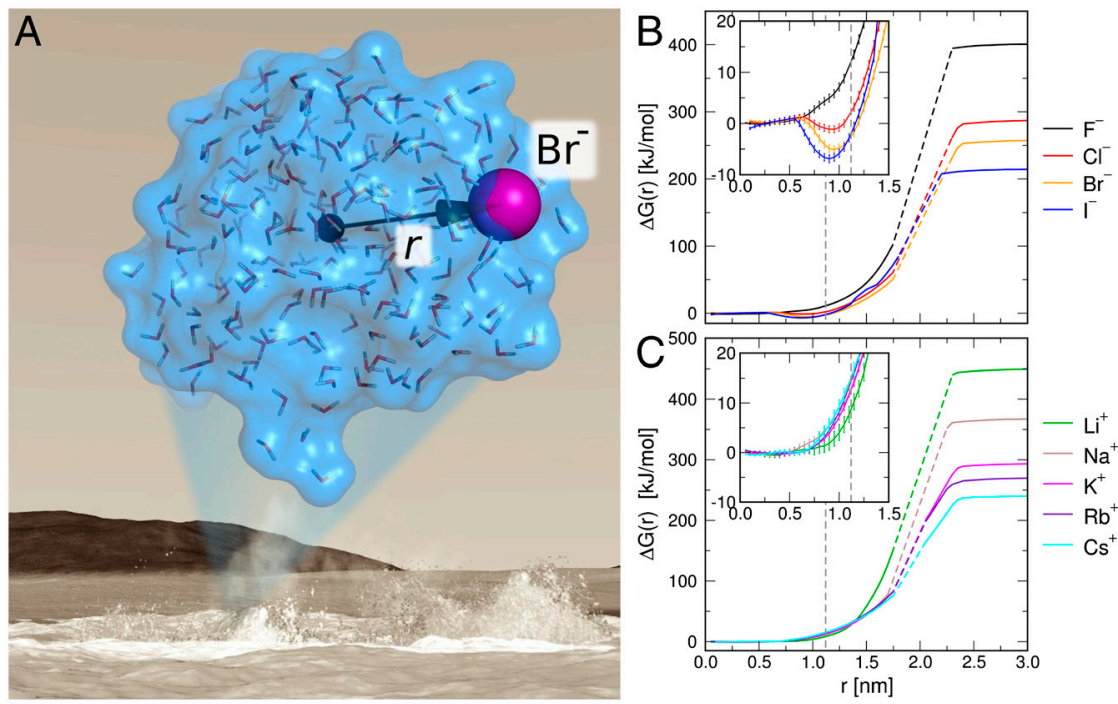

Fig. 1. (A) Simulation snapshot of a bromide ion (purple sphere) partially dehydrated at the surface of a water droplet (blue surface). The arrow indicates the distance $r$ of the ion to the center of mass of the droplet (black sphere). Free-energy profiles $(\Delta G(r))$ of $(B)$ halide anions and $(C)$ alkali cations for moving the ion from the droplet interior past the droplet surface into vacuum. All free-energy profiles are defined to zero in the droplet interior. The profiles for $\mathrm{Cl}^{-}$, $\mathrm{Br}^{-}$, and $I^{-}$show a minimum at the droplet surface $(A$, inset, $r \approx 1.0 \mathrm{~nm})$, indicating the surface preference for these ions. In contrast, the profiles for $\mathrm{F}^{-}$and the alkali cations monotonically increase with $r$, demonstrating that full solvation of these ions is energetically more favorable. At the surface of the droplet (indicated approximately by the gray dashed lines) frequent jumps of water molecules from the droplet onto the ion leads to a very slowly converging mean force and typically to an underestimation of the overall solvation free energy. To yield continuous free-energy profiles, we have therefore computed the solvation free energy for all ions using thermodynamic integration, and scaled the free-energy profiles between $r=1.7 \mathrm{~nm}$ and $r=2.2 \mathrm{~nm}$ thereafter (dashed line).

Fig. $3 A$ and $B$ shows the enthalpic component $\Delta H(r)$, and in Fig. $3 C$ and $D$ the entropic component $T \Delta S(r)$ of the free energy of solvation. Note that these components sum up to the PMF via $\Delta G(r)=\Delta H(r)-T \Delta S(r)$. Remarkably, the enthalpies for all halide anions display a minimum at the droplet surface (referred to as region II in Fig. 2). For $\mathrm{Cl}^{-}, \mathrm{Br}^{-}$, and $\mathrm{I}^{-}$, this enthalpic minimum is only partially compensated for by a reduced entropy on the surface as compared to the bulk (Fig. 3C). Hence, the surface preference of the large halide anions is based on an enthalpic effect. For $\mathrm{F}^{-}$, the smallest halide anion, the entropic component overcompensates the slight enthalpic minimum at the surface, summing up to a monotonically increasing PMF. Hence $\mathrm{F}^{-}$ions are driven from the surface into the droplet by an effective entropic force. The alkali cations show monotonically increasing PMFs as well (Fig. $1 C$ ), which is entirely due to the enthalpic term $\Delta H(r)$ (Fig. $3 B)$. Entropic effects do not seem to play a role in the bulk preference of alkali cations, because the entropic profiles (Fig. $3 D$ ) are nearly flat inside the droplet.
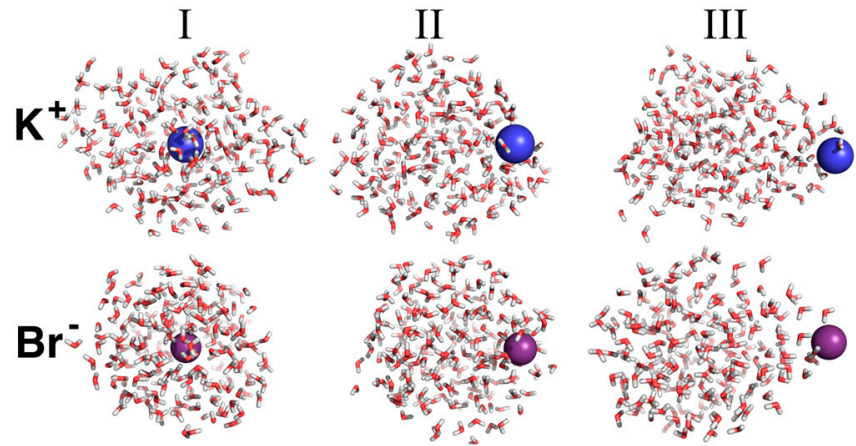

Fig. 2. Characteristic trajectory snapshots of $\mathrm{K}^{+}$and $\mathrm{Br}^{-}$ions in a water droplet. Region I: ion at the center of mass of the water droplet. Region II: ion $0.9 \mathrm{~nm}$ from center of mass of the water droplet. This region is the energetically most favorable for the halide anions. Region III: ion $1.4 \mathrm{~nm}$ from the center of mass of the droplet. Here all ions experience a force pulling them back into the water. Compare the three regions to Fig. 3. Movies S1 and S2 show the ions being pulled out of the droplets.
By decomposing the enthalpic component $\Delta H(r)$ into waterwater and water-ion interactions, represented by the time averaged interaction potentials (Fig. $3 E-H$ ), we can pin down the factors that govern surface/bulk preference of ions. The potential energies are plotted relative to the center of the droplet (i.e., $V(r=0) \equiv 0$ in all cases). In this manner, the water-water and the ion-water interactions sum up to the enthalpic plots in Fig. $3 A$ and $B$. For the halide anions, the water-water interaction is more favorable (more negative) by several tens of $\mathrm{kJ} / \mathrm{mol}$ if the ion is partially desolvated, as compared to a fully solvated ion (Fig. 3E). As expected, some ion-water interaction is lost upon partial desolvation (Fig. $3 G$ ), but the gain in water-water interaction outweighs the loss in ion-water interaction, yielding the minima in the $\Delta H(r)$ curves around $r=1 \mathrm{~nm}$ in Fig. $3 A$. Only for larger $r>1.5 \mathrm{~nm}$ - that is, when the ions start to become fully desolvated-does the loss in ion-water interaction outweigh the gain in water-water interaction, leading to the unfavorable sharp increase in $\Delta H(r)$. Compared to the halide anions, the solvation energetics of the alkali cations are qualitatively different. Here, the water-water interaction is almost unaffected by a partial desolvation of the ions (Fig. $3 F$ ). Instead, the monotonic loss of ion-water interaction (Fig. $3 H$ ) accounts for the monotonically increasing $\Delta H(r)$ curves and, hence, also for the bulk preference of the alkali cations.

Is there a structural explanation for the shape of the enthalpic and entropic curves in Fig. 3? Experiments suggest that water-ion interactions are highly localized in space. Femtosecond pumpprobe spectroscopy demonstrated that sulfate and perchlorate ions do not influence the rotational dynamics of water molecules outside the first solvation shells of the ions (25), indicating that the insertion of these ions does not affect the hydrogen bond network on a larger scale. Similar findings were reported for the solvation of alkali cations based on O-H vibrational spectroscopy (26). It has, however, also been reported that at higher concentrations some ions can have strong nonadditive effects on water dynamics (27), obscuring the simpler picture of Omta et al. (25). In an attempt to find structural explanations for the energetic curves (Fig. 3), we have computed radial distribution functions (RDFs) for water molecules around the ions and around other 

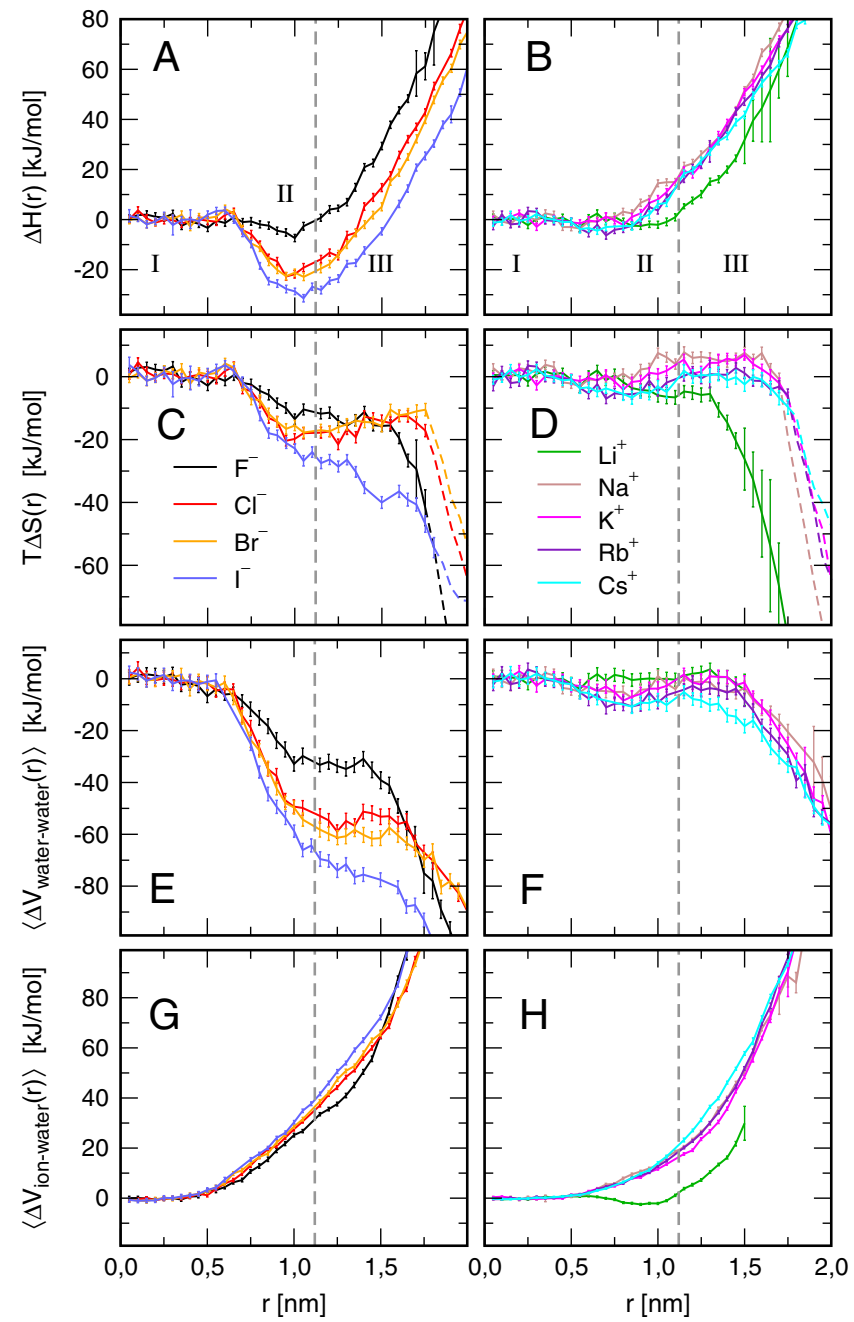

Fig. 3. Decomposition of the potentials of mean force into enthalpic components $(A$ and $B$ ) and entropic components $(C$ and $D$ ), as well as decomposition of the enthalpic component into $(E$ and $F$ ) water-water and ( $G$ and $H$ ) ion-water interactions. The entropy and enthalpy are plotted as the energy difference with respect to the droplet interior. $(A)$ The surface preference of $\mathrm{Cl}^{-}, \mathrm{Br}^{-}$, and $\mathrm{I}^{-}$is induced by a substantial minimum in the enthalpic component $\Delta H(r)$ near the droplet surface $(r \approx 1 \mathrm{~nm})$, which is only partially compensated by a lower entropy $(C)$. In contrast, for $\mathrm{F}^{-}$on the droplet surface, the substantial loss of entropy ( $C$, black curve) is only partly compensated by a slightly decreased enthalpy ( $A$, black curve), indicating that an entropic driving force explains the bulk preference of $\mathrm{F}^{-}$. (B) Alkali cations are in the droplet bulk due to the favorable enthalpy. Partly desolvated halide anions on the droplet surface allow for more favorable water-water interactions $(E)$. Alkali cations are driven into the bulk by more favorable ionwater interactions $(H)$.

water molecules (Figs. S1 and S2 in SI Appendix). We found that the changes in the ion-water RDF as the ions move from bulk to surface is identical for all ions-the changes merely reflect the gradual desolvation of the ions. The water-water RDFs are virtually independent of the ion position in all cases. The waterion structure can be examined by correlating the features of the PMFs (Fig. $1 B$ and $C$ ) with order parameters representing the hydration structure around the ions. To this end, we have calculated two-dimensional energy landscapes with distance $r$ on one axis and either the ionic coordination number or the orientation of water in the first solvation shell with respect to the ion on the other axis (Figs. S3 and S4 in SI Appendix). Neither of these analyses yields a simple mechanistic explanation for the bulk/surface preference. This indicates that the structural rearrangements underlying changes in enthalpy are very subtle.
Specific ion properties such as the polarizability and the size of the ions have been proposed to explain the bulk versus surface preference (for reviews see refs. 10 and 11). In particular, it has been suggested that the surface potential at the water-air interface induces a dipole in polarizable anions, whereas the ion is not polarized in the bulk. Consequently, the induced dipole of the ion would be attracted by the air-water interface, compensating for the loss of solvation as the ions move to the surface. Our results do not support this view, because the ion-water interaction becomes less favorable as the halide anions move to the surface (Fig. 3G). Remarkably, the loss in ion-water interaction is most prominent for the highly polarizable $\mathrm{I}^{-}$, demonstrating that the water molecules do not attract the ion to the surface. To shed more light on the putative effect of ion polarization, Fig. $4 \mathrm{~A}$ and $B$ presents the average ion dipole as a function of position. The polarization of the halide ions increases only slightly as the ions move to the surface (Fig. $4 A$ ), but this slight increase is in fact strongest for $\mathrm{F}^{-}$, which does not display any surface preference. In addition, Fig. $4 C$ and $D$ shows the average dipole of water molecules as a function of ion position. Interestingly, as halide anions move to the surface, the water dipoles increase simultaneously with more favorable water-water interaction (Fig. $3 E$ ), which explains how the water-water interaction energy can become stronger without changing the water-water structure. However, $\mathrm{F}^{-}$shows a water dipole curve similar to the large halide anions, despite its qualitatively different bulk preference. Thus, we must conclude that neither the ion- nor the water-polarization can provide a complete explanation for the bulk/surface preference. To assess if polarizability in combination with the size of the ion could explain the bulk/surface preference, we have computed PMFs for hypothetical cations with size and polarizability taken from the halide ions but with an inverted (positive) charge (Fig. S5 in SI Appendix). In contrast to the large halide ions, none of these hypothetical ions show any surface preference. This demonstrates that the specific polarizability/size combination of halide ions is not sufficient to determine the bulk/surface preference either, because the sign of the charge plays an important role as well. This notion rules out the possibility to fully explain the surface preference based on continuum models that do not explicitly include the asymmetry of the water molecules.

Our simulations demonstrate that the source of the bulk/ surface preference is different for different ions and that simple models and approximations may have to be abandoned in favor of the quantitative energetic description of solvation presented here. The simulations suggest that no single mechanistic explanation exists that elucidates the bulk/surface preference of all the alkali and halide ions. In fact, structural changes must be inter-
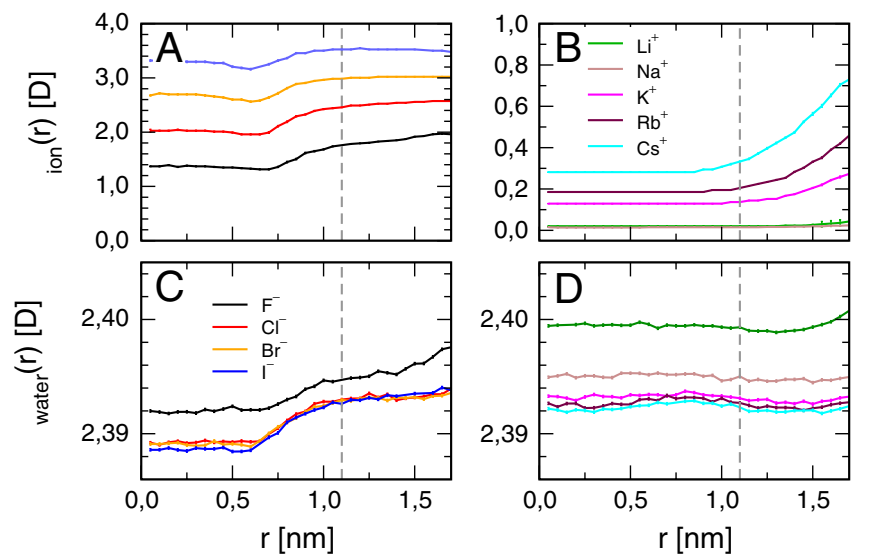

Fig. 4. Ion dipole as a function of ion position for $(A)$ halide anions and $(B)$ alkali cations (with respect to the center of charge). Average dipole of water molecules as a function of ion position for $(C)$ halide anions and (D) alkali cations. Errors in the figures are less than $1 \%$. 
preted with care. For instance, the two-dimensional energy landscape for $\mathrm{I}^{-}$as a function of $r$ and water orientation (Fig. S4 in SI Appendix) indicates higher configurational freedom of water when $\mathrm{I}^{-}$is located at the surface. This could lead to the false conclusion that the surface preference of $\mathrm{I}^{-}$is due to higher water entropy. However, our energetic analysis clearly demonstrates that, on the contrary, $\mathrm{I}^{-}$on the surface is entropically unfavorable (Fig. $3 C$, blue curve).

The energetics of partial ion desolvation, which govern the bulk versus surface preference of ions, are significantly more complex than what was appreciated previously. Recall that the large free-energy differences for moving ions from vacuum to bulk are dominated by the favorable ion-water interaction. In contrast, the bulk versus surface preferences are based on a fine balance between entropic and enthalpic driving forces, where the latter are in turn based on competing ion-water and water-water interactions. The surface preference for the highly polarizable halide anions $\mathrm{Cl}^{-}, \mathrm{Br}^{-}$, and $\mathrm{I}^{-}$was found to be enthalpically driven, because partly desolvated halide anions allow for more favorable water-water interactions compared to fully solvated halide anions. The $\mathrm{F}^{-}$bulk preference is due to an entropic effect, whereas the alkali cations are driven into the bulk by the highly favorable ion-water interactions. It would be very interesting if the experiments of Onorato et al. (19) on $\mathrm{Br}^{-}$were repeated at different temperatures (and, obviously, with $\mathrm{Cl}^{-}$and $\mathrm{I}^{-}$) in order to be able to decompose the surface affinity into entropic and enthalpic components, as this would yield a route to verify the results in Fig. 3 in this work.

The fundamental properties of aerosols remain among the largest uncertainties in climate science (6). By now, it must be excruciatingly clear that the properties of aerosols are determined by a complex interplay of enthalpy and entropy: Approximations and simplified models can give results that are both quantitatively and qualitatively incorrect (28). The fact that the effects of salts can be nonadditive complicates matters further (27). We have shown here that atomistic simulations that include polarization explicitly can unravel the intricate energetics of ion solvation and, with this, enhance understanding of aerosols.

\section{Materials and Methods}

Potentials of Mean Force. The PMFs $(\Delta G(r))$ were computed based on a protocol by Johansson and Lindahl (29). Along the reaction coordinate $r$ that is, the distance of the ion from the center of mass (COM) of the water droplet-101 positions were selected in the range $0.05 \mathrm{~nm} \leq r \leq 5 \mathrm{~nm}$, with a distance of $0.05 \mathrm{~nm}$ between adjacent positions. For each position, the respective ion was placed into the structure and the distance between ion and droplet COM was constrained for the following simulations. After energy minimization, systems in the range of $r \leq 2.25 \mathrm{~nm}$ were simulated for $5 \mathrm{~ns}$, and the other systems for $100 \mathrm{ps}$. For each $r$ value, the mean force on the ion was computed after removing the first 50 ps of each trajectory for equilibration, and the PMF was computed by integration of the mean force. Statistical errors for all properties were computed by binning analysis (30), and the errors in the PMFs using standard error propagation. An analytical entropy correction for spherical systems was applied to the PMFs (31), according to:

$$
\Delta S_{r_{0}}(r)=k_{\mathrm{B}} \ln \left(\frac{r}{r_{0}}\right)^{2}
$$

where $k_{\mathrm{B}}$ is the Boltzmann factor and $r_{0}$ was taken to be $0.35 \mathrm{~nm}$ where the $\mathrm{PMF}$ is near zero.

lons that are located a few tenths of a nanometer above the droplet surface $(1.7 \mathrm{~nm} \leq r \leq 2.2 \mathrm{~nm})$ frequently induce jumps of water molecules from the droplet onto the ion, leading to a very slowly converging mean force and typically to an underestimation of the overall solvation free energy. In contrast, the mean forces converged rapidly for $r<1.7 \mathrm{~nm}$ and $r>2.2 \mathrm{~nm}$. To yield continuous PMFs, we have therefore computed the solvation free

1. Conover JH (1966) Anomalous cloud lines. J Atmos Sci 23:778-785.

2. Finlayson-Pitts $\mathrm{BJ}$ (1983) Reaction of $\mathrm{NO}_{2}$ with $\mathrm{NaCl}$ and atmospheric implications of $\mathrm{NOCl}$ formation. Nature 306:676-677. energy $\Delta G_{\mathrm{Tl}}=G\left(r_{1}\right)-G\left(r_{2}\right)$ for all ions using thermodynamic integration (TI) (32), where we chose $r_{1}=0.6 \mathrm{~nm}$ and $r_{2}=5.0 \mathrm{~nm}$. The PMFs were then corrected in the range $1.7 \mathrm{~nm} \leq r \leq 2.2 \mathrm{~nm}$, such that the free energy between $r_{1}$ and $r_{2}$ in the PMF is in agreement with the TI calculation (dashed lines in Fig. 1). $\Delta G_{\mathrm{TI}}$ was computed in four steps:

i. Turn off Coulomb interactions between ion and water for an ion restrained at $r_{1}$.

ii. Turn off in addition the Lennard-Jones (LJ) interactions between ion and water for the ion restrained at $r_{1}$.

iii. Calculate analytically the increase in entropy for moving a noninteracting particle from $r_{1}$ to $r_{2}$, given by Eq. 1 .

iv. Turn on Coulomb and $\mathrm{LJ}$ interactions for the ion restrained at $r_{2}$.

Steps i, ii, and iv were computed using an alchemical reaction coordinate $\lambda$, where $\lambda=0$ and $\lambda=1$ correspond to the initial and final state, respectively. Steps i, ii, and iv were decomposed into 21,21 , and 11 equally spaced $\lambda$-steps, respectively, and the systems were simulated for 300,300 , and 100 ps at each $\lambda$-value, respectively. The free-energy differences for steps i, ii, and iv were subsequently calculated by integrating $\langle\partial \mathscr{H} / \partial \lambda\rangle$ from $\lambda=0$ to $\lambda=1$. Here, $\langle\cdot\rangle$ denotes the average computed from the respective trajectory, after removing the first 50 ps for equilibration, and $\mathscr{H}$ the Hamiltonian of the system.

Enthalpies $\Delta H(r)$ (Fig. 3) were computed by averaging the potential energy over the respective simulation trajectory. The kinetic energy cancels from these curves because we show only the difference of the enthalpy with respect to the ion being in the droplet interior. Entropies were computed via $T \Delta S(r)=\Delta H(r)-\Delta G(r)$. The water-water interaction energies were computed by averaging the sum of the $\mathrm{LJ}$ and Coulomb potentials between water molecules plus the water-polarization energy. Likewise, ion-water interaction energies were computed by averaging the sum of the $\mathrm{LJ}$ and Coulomb potentials between the ion and all water molecules plus the ion polarization energy.

Statistical errors for $\Delta G_{\mathrm{Tl}}$ were $\leq 1.8 \mathrm{~kJ} / \mathrm{mol}$. The uncertainties in the PMFs and in the energetic curves are indicated as error bars in Figs. 3 and 4 , corresponding to one standard deviation.

Force Field and Simulation Parameters. Accurate simulations of ion solvation require polarizable force fields $(12,16,33-35)$. Here we applied the polarizable models of Lamoureux et al. for water (SWM4-DP) (20) and for ions (21); these ion models were parameterized in conjunction with the SWM4-DP water model. The parameters in these models were optimized to reproduce the binding energies of gas-phase monohydrates and the hydration free energies in the bulk liquid. These models have since been updated and extended with additional ions (36), yielding confidence that further computational investigations on airborne chemicals can be done in order to advance knowledge of atmospheric chemicals and their interactions.

All simulations were performed in double precision using the GROMACS software $(37,38)$. Newton's equation of motion was solved using the Leap-Frog integration scheme (39) with a time step of $2 \mathrm{fs}$. No cutoffs were applied to $\mathrm{LJ}$ or Coulomb interactions.

The SETTLE algorithm was used to constrain the water molecules (40). During the PMF calculations, the temperature was controlled at $293.15 \mathrm{~K}$

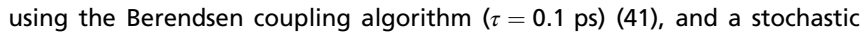
dynamics integrator (42) was applied for the TI calculation ( $\tau=0.1 \mathrm{ps})$. Because we simulated at room temperature, individual water molecules would frequently evaporate from the droplet surface, which would lead to an illdefined droplet COM. To avoid such evaporation, we applied a spherical flat-bottom quadratic potential acting on the water. That potential was implemented as an additional force $F(r)=-k / 2\left(r-r_{\mathrm{fb}}\right)^{2} H\left(r-r_{\mathrm{fb}}\right)$ pointing toward the COM of the droplet, where $k=1,000 \mathrm{~kJ} \mathrm{~mol}^{-1} \mathrm{~nm}^{-1}$ denotes the force constant, $r_{\mathrm{fb}}=1.4 \mathrm{~nm}$ the radius of the sphere around the COM without any additional force, and $H$ the Heaviside step function.

The Drude (shell) particles were minimized at each time step until the root-mean-square force on these particles was $\leq 0.001 \mathrm{~kJ} / \mathrm{mol}(43)$.

ACKNOWLEDGMENTS. The authors thank N. Tîmneanu, A. Johansson, J. Åqvist, and $M$. van Lun for helpful discussions. The Helmholtz Association is thanked for supporting C.C. through the Center for Free Electron Laser Research. The Swedish Research Council is acknowledged for financial support to D.v.d.S. (2007-5671) and for a grant of computer time (SNIC 022/09-10). J.S.H. acknowledges support through a Marie-Curie Intra-European fellowship within the 7th European Community framework program.

3. Finlayson-Pitts BJ, Ezell MJ, Pitts JN, Jr (1989) Formation of chemically active chlorine compounds by reactions of atmospheric $\mathrm{NaCl}$ particles with gaseous $\mathrm{N}_{2} \mathrm{O}_{5}$ and $\mathrm{ClONO}_{2}$. Nature 337:241-244. 
4. Laskin A, et al. (2003) Reactions at interfaces as a source of sulfate formation in sea-salt particles. Science 301:340-344.

5. Laskin A, et al. (2006) A new approach to determining gas-particle reaction probabilities and application to the heterogeneous reaction of deliquesced sodium chloride particles with gas-phase hydroxyl radicals. J Phys Chem A 110:10619-10627.

6. Finlayson-Pitts BJ (2009) Reactions at surfaces in the atmosphere: Integration of experiments and theory as necessary (but not necessarily sufficient) for predicting the physical chemistry of aerosols. Phys Chem Chem Phys 11:7760-7779.

7. Knipping EM, et al. (2000) Experiments and simulations of ion-enhanced interfacial chemistry on aqueous nacl aerosols. Science 288:301-306.

8. Jungwirth P, Tobias DJ (2000) Surface effects on aqueous ionic solvation: A molecular dynamics simulation study of $\mathrm{NaCl}$ at the air/water interface from infinite dilution to saturation. J Phys Chem B 104:7702-7706.

9. Ghosal S, et al. (2005) Electron spectroscopy of aqueous solution interfaces reveals surface enhancement of halides. Science 307:563-566.

10. Petersen P, Saykally R (2006) On the nature of ions at the liquid water surface. Annu Rev Phys Chem 57:333-364.

11. Jungwirth P, Tobias DJ (2006) Specific ion effects at the air/water interface. Chem Rev 106:1259-1281.

12. Caleman C, van der Spoel D (2007) Evaporation from water clusters containing singly charged ions. Phys Chem Chem Phys 9:5105-5111.

13. Liu D, Ma G, Levering LM, Allen HC (2004) Vibrational spectroscopy of aqueous sodium halide solutions and air-liquid interfaces: Observation of increased interfacial depth. $J$ Phys Chem B 108:2252-2260.

14. Ottosson N, et al. (2010) The influence of concentration on the molecular surface struc ture of simple and mixed aqueous electrolytes. Phys Chem Chem Phys 12:10693-10700.

15. Kester DR, Duedall IW, Conners DN, Pytkowicz RM (1967) Preparation of artificial seawater. Limnol Oceanogr 12:176-179.

16. Yoo S, Lei YA, Zeng XC (2003) Effect of polarizability of halide anions on the ionic solvation in water clusters. J Chem Phys 119:6083-6091.

17. Herce DH, Perera L, Darden TA, Sagui C (2005) Surface solvation for an ion in a water cluster. J Chem Phys 122:024513.

18. Noah-Vanhoucke J, Geissler PL (2009) On the fluctuations that drive small ions toward and away from, interfaces between polar liquids and their vapors. Proc Natl Acad Sc USA 106:15125-15130.

19. Onorato RM, Otten DE, Saykally RJ (2010) Measurement of bromide ion affinities for the air/water and dodecanol/water interfaces at molar concentrations by UV second harmonic generation spectroscopy. J Phys Chem C 114:13746-13751.

20. Lamoureux G, MacKerell AD, Roux B (2003) A simple polarizable model of water based on classical drude oscillators. J Chem Phys 119:5185-5197.

21. Lamoureux G, Roux B (2006) Absolute hydration free energy scale for alkali and halide ions established from simulations with a polarizable force field. J Phys Chem B 110:3308-3322.

22. Yang Z-Z, Li X (2005) lon solvation in water from molecular dynamics simulation with the ABEEM/MM force field. J Phys Chem A 109:3517-3520.
23. Brown MA, et al. (2008) Ion spatial distributions at the liquid-vapor interface of aqueous potassium fluoride solutions. Phys Chem Chem Phys 10:4778-4784.

24. Ahadi E, Konermann L (2010) Surface charge of electrosprayed water nanodroplets: A molecular dynamics study. J Am Chem Soc 132:11270-11277.

25. Omta AW, Kropman MF, Woutersen S, Bakker HJ (2003) Negligable effect of ions on the hydrogen-bond structure in liquid water. Science 301:347-349.

26. Smith JD, Saykally RJ, Geissler PL (2007) The effects of dissolved halide anions on hydrogen bonding in liquid water. J Am Chem Soc 129:13847-13856.

27. Tielrooij KJ, Garcia-Araez N, Bonn M, Bakker HJ (2010) Cooperativity in ion hydration. Science 328:1006-1009.

28. Tobias DJ, Hemminger JC (2008) Chemistry—getting specific about specific ion effects. Science 319:1197-1198.

29. Johansson ACV, Lindahl E (2008) Position-resolved free energy of solvation for amino acids in lipid membranes from molecular dynamics simulations. Protein 70:1332-1344.

30. Hess B (2002) Determining the shear viscosity of model liquids from molecular simulation. J Chem Phys 116:209-217.

31. Neumann RM (1980) Entropic approach to brownian movement. Am J Phys 48:354-357

32. Kirkwood JG (1935) Statistical mechanics of fluid mixtures. J Chem Phys 3:300-313.

33. Stuart SJ, Berne BJ (1996) Effects of polarizability on the hydration of the chloride ion. J Phys Chem 100:11934-11943.

34. Carignano MA, Karlström G, Linse P (1997) Polarizable ions in polarizable water: A molecular dynamics study. J Phys Chem B 101:1142-1147.

35. Chang TM, Dang LX (2006) Recent advances in molecular simulations of ion solvation at liquid interfaces. Chem Rev 106:1305-1322.

36. $\mathrm{Yu} \mathrm{H}$, et al. (2010) Simulating monovalent and divalent ions in aqueous solution using a drude polarizable force field. J Chem Theory Comput 6:774-786.

37. van der Spoel D, et al. (2005) GROMACS: Fast, flexible and free. J Comput Chem 26:1701-1718.

38. Hess B, Kutzner C, van der Spoel D, Lindahl E (2008) Gromacs 4.0: Algorithms for highly efficient, load-balanced, and scalable molecular simulation. J Chem Theory Comput 4:435-447.

39. Berendsen HJC, van Gunsteren WF (1986) Practical algorithms for dynamics simulations. Molecular-Dynamics Simulation of Statistical-Mechanical Systems, eds G Ciccotti and WG Hoover (North-Holland, Amsterdam).

40. Miyamoto S, Kollman PA (1992) SETTLE: An analytical version of the SHAKE and RATTLE algorithms for rigid water models. J Comput Chem 13:952-962.

41. Berendsen HJC, Postma JPM, DiNola A, Haak JR (1984) Molecular dynamics with coupling to an external bath. J Chem Phys 81:3684-3690.

42. van Gunsteren WF, Berendsen HJC (1988) A leap-frog algorithm for stochastic dynamics. Mol Simulat 1:173-185.

43. van Maaren PJ, van der Spoel D (2001) Molecular dynamics simulations of water with a novel shell-model potential. J Phys Chem B 105:2618-2626. 